\title{
Changes in pelvic organ prolapse surgery
}

\author{
Elise De, MD \\ The Urological Institute of NENY, Albany, NY
}

See related article on page 100 .

Cite as: Can Urol Assoc J 2014;8(3-4):107-8. http://dx.doi.org/10.5489/cuaj.2082

Published online April 14, 2014.

$\mathrm{T}$ he current study updates the data on trends in pelvic organ prolapse surgery in the United States, comparing robotic-assisted vaginal vault suspension (RAVVS) to open vaginal vault suspension (OVVS). ${ }^{1}$ The authors look at outcomes and utilization in the Nationwide Inpatient Sample (NIS) from 2009 to 2010. The analysis shows an increase in utilization of RAVVS over time, lower blood loss, higher intraoperative complications, and higher charges, but equivalent overall postoperative complications.

Large population-based studies, in my mind, do more to raise questions than to answer them. However, there is great value in raising the questions.

Any assessment of robotic outcomes needs to take into account the stage of familiarity of the surgeon. The fact that utilization was still increasing during the 1-year sample implies that RAVVS was still being adopted by some of the surgeons sampled. The advantages of the NIS include large sample size and a broad representation of practice type. However, it is not possible to distinguish results from high volume surgeons later on the learning curve from lower volume surgeons or those newer to the technology. The annual caseload at the centres performing RAVVS was higher, but this was not broken down by surgeon - the higher volume centre might have been more likely to purchase a robot or to hire an additional newer surgeon. The authors comment that the higher intraoperative complication rate may be attributable to the learning curve. The perioperative complications captured were injury to organ nerve or vessel, transfusion, death, prolonged length of stay, elevated hospital charges, cardiac, wound, vascular, genitourinary, neurological, infectious, and miscellaneous complications, and death. More

subtle indicators of surgeon comfort, such as intraoperative consultation, presence of robotic representative in the room, conversion and console time, are not measurable with this type of data. Since prolonged case time may lead to rhabdomyolysis or positioning injury and increased costs, important qualifiers may be missing. Other factors are at play as well. Patients who had RAVVS were younger, more likely to have private insurance (means), and had fewer comorbidities, which might lead to a shorter hospital stay. Those who had OVVS may have had multiple prior surgeries leading to the decision against a robotic approach. Probably the most significant limitation of this study, as recognized by the authors, is that the NIS does not distinguish between the vaginal and abdominal approaches to vault suspension. Therefore the RAVVS is being compared to a heterogeneous group rather than its open counterpart.

Having myself learned to perform RAVVS post-fellowship 7 years ago, I have opinions regarding the factors playing into the data above. The learning curve is a significant factor for patient outcome and it is important to maintain experienced proctors, surgical assists, circulators, and residents to protect against complications. I now strongly prefer the RAVVS to open abdominal sacrocolpopexy due to the ease of dissection, precision, visibility, and postoperative pain. However, I still perform vaginal approaches with regularity and open abdominal approaches occasionally based on the patient scenario, stage of prolapse and prior surgeries. The considerations in patient selection are sure to confuse outcomes in a large population utilization study.

Despite all the qualifiers, the trends shown in this study do make sense. Lower blood loss, shorter hospital stay, fewer wound complications in RAVVS have been suggested by prior smaller studies. The authors have shown trends that raise important questions and they themselves recommend prospective studies to further elucidate on the questions raised. Comparison of RAVVS to open abdominal sacrocolpopexy in a randomized fashion among experi- 
De

enced surgeons would give the purest information regarding outcomes.

Competing interests: Dr. De declares no competing financial or personal interests.

\section{Reference}

1. Li H, Sammon J, Roghmann F, et al. Utilization and perioperative outcomes of robotic vaginal vault suspension compared to abdominal or vaginal approaches for pelvic organ prolapse. Can Urol Assoc $\mathrm{J}$ 2014;8:100-6. http://dx.doi.org/10.5489/cuaj.1858

Correspondence: Dr. Elise De, The Urological Institute of NENY, Albany, NY 12208; elisede@gmail.com

\section{CUAJ JAUC}

We are pleased to now offer online manuscript and review submission for CUAJ. As of January 1, 2013, $\mathrm{CUAJ}$ is only receiving papers through its online manuscript submission system.

Please take a moment to register. Registration is free and quick!

When you register, you will be able to:

- Register as an Author, Reader and Reviewer

- Check the status of your paper online 24 hours a day/7 days a week

- Submit papers anytime

- Easily access papers for review

- Get immediate access to any job opportunities and news

To register:

1. Go to http://journals.sfu.ca/cuaj/index.php/journal/user/register

2. Register yourself as an author (and reviewer if you want to review papers for CUAJ)

3. Once you are registered, you will receive an email with your username and password - these will give you access to the system and allow you access to the status of your article

To submit a paper:

1. Go to http://journals.sfu.ca/cuaj/index.php/journal/index

2. Using the LOGIN tab, enter your username and password (sent to you in an email)

3. Go to the Author tab and click on the "Start your submission" link

4. Follow the 5-step submission process

- $\quad$ Make sure you submit a blinded copy of the paper for review. A blinded paper means that any identifying information should be deleted from the paper (i.e., your name, institution, contact information).

- $\quad$ Your tables and figures should be submitted as supplementary files.

- $\quad$ Before you upload files to the system, be sure they are not open on your system (this may cause problems in uploading).

Once your paper is submitted, you can follow its track through our editorial process - please keep your username and password handy.

At the moment, you can still access older issues of CUAJ at www.cuaj.ca - however, within the next few months, we will be migrating all our past issues to this new site (while keeping the cuaj.ca domain name). If you have any questions, please email me. I would be happy to walk you through the process.

Josephine Sciortino, CUA Corporate Office

185 Dorval Avenue, Suite 401, Dorval, QC H9S 5J9

Tel: 514-395-0376, ext. 40; Fax: 514-395-1664

Email: josephine.sciortino@cua.org 